\title{
A UNIFIED VIEW OF CONVECTIVE TRANSPORTS BY STRATOCUMULUS CLOUDS, SHALLOW CUMULUS CLOUDS, AND DEEP CONVECTION
}

\author{
David A. Randall \\ Department of Atmospheric Science \\ Colorado State University \\ Fort Collins, Colorado 80523
}

\section{Introduction}

We have developed a bulk PBL model with a simple internal vertical structure and a simple second-order closure, designed for use as a PBL parameterization in a large-scale model. The model allows the mean fields to vary with height within the PBL, and so must address the vertical profiles of the turbulent fluxes, going beyond the usual "mixed-layer" assumption that the fluxes of conservative variables are linear with height. This is accomplished using the same convective mass flux approach that has also been used in cumulus parameterizations. The purpose of this brief paper is to show that such a mass flux model can include, in a single framework, the "compensating subsidence" concept, downgradient mixing, and well-mixed layers.

\section{Generalized mass flux model}

The "convective mass flux" concept introduced by Arakawa has been used in a variety of modeling and observational studies, most directed either at deep cumulus convection or at stratocumulus convection; the connections between the two applications have not been made previously.

For an ascending region with fracitonal area $\sigma$, the conservation equations for mass and an arbitrary scalar $\psi$ can be written as

$$
\begin{gathered}
\frac{\partial}{\partial t}(\rho \sigma)=-\sigma \nabla \cdot(\rho \overline{\mathbf{V}})+\rho(\mu-v)-\rho \frac{\partial}{\partial p}\left(\omega_{u} \sigma\right) \\
\frac{\partial}{\partial t}\left(\rho \sigma \psi_{u}\right)=-\sigma\left\{\nabla \cdot(\rho \overline{\mathbf{V} \psi})+\nabla \cdot\left[\rho \overline{\mathbf{V}}\left(\psi_{u}-\bar{\psi}\right)\right]\right\}+\rho\left(\mu \psi_{d}-v \psi_{u}\right) \\
-\rho \frac{\partial}{\partial p}\left(\omega_{u} \sigma \psi_{u}\right)-\rho \tau_{d i s}^{-1} \sigma\left(\psi_{u}-\bar{\psi}\right)+\rho \sigma\left[g \frac{\partial f_{\psi, u}}{\partial p}+S_{\psi, u}\right]
\end{gathered}
$$


for the ascending parcels. Similar equations can be written for the descending regions. (2.2) generic variable $\psi$ represents an intensive therme or the the mix or the the mixing ratio of water. Other notation is as follows: $\rho$ is the density; $\mathbf{V}$ is the horizontal velocity vector; $\omega=-\rho g w$, where $w$ is the vertical velocity, and $g$ is the acceleration of gravity; and $\mathrm{p}$ is pressure. The air entering rising parcels has been assumed to have the average properties of the sinking parcels, and vice versa. Area averages satisfy

$$
(-)=()_{u} \sigma+()_{d}(1-\sigma)
$$

where an overbar denotes an area average (over a grid box, say); this notation will be used only where necessary to avoid confusion. Subscripts $u$ and denote upward and downward moving parcels, respectively. We can interpret $\mu^{-1}$ and $v^{-1}$ as time scales for mass flow from downdrafts into updrafts, and from updrafts into downdrafts, respectively. The vertical turbulent flux of $\psi$ due to small eddies is denoted by $f_{\psi}$. The small-eddy fluxes are logically necessary near the top and bottom of the PBL, where the organized vertical motions associated with the convective circulations must vanish. The time scale for destruction of the convective circulations by lateral mixing due to smaller-scale turbulence is $\tau_{\text {dis. }}$. The source of $\psi$ per unit mass per unit time is denoted by $S_{\Psi}$. This could represent, for example, the effects of radiation.

From (2.1) and (2.2) and the correponding "downdraft" equations, and using (2.3), we canrecover the area-averaged conservation equations for mass and $\psi$ :

$$
\begin{gathered}
\frac{\partial}{\partial t} \rho=-\nabla \cdot(\rho \overline{\mathrm{V}})-\rho \frac{\partial \bar{\omega}}{\partial p} \\
\frac{\partial}{\partial t}(\rho \bar{\psi})=-\nabla \cdot(\rho \overline{\mathrm{V} \psi})-\rho \frac{\partial}{\partial p}\left[\bar{\omega} \bar{\psi}-g\left(\mathrm{~F}_{\psi}+f_{\psi}\right)\right]+\rho \bar{S}_{\psi}
\end{gathered}
$$

Naturally, all terms involving $\mu, v$, and $\tau_{\text {dis }}$ have dropped out of (2.4-5). In (2.5), the turbulent fluxes associated with the convective circulations are represented by

$$
\begin{aligned}
g \boldsymbol{J}_{\psi}=-\overline{\omega^{\prime} h^{\prime}} & =-\left[\left(\omega_{k}-\bar{\omega}\right)\left(\psi_{k}-\bar{\psi}\right) \sigma+\left(\omega_{d}-\bar{\omega}\right)\left(\psi_{d}-\bar{\psi}\right)(1-\sigma)\right] \\
& =-\omega_{\cdot}\left(\psi_{k}-\psi_{d}\right),
\end{aligned}
$$

where

$$
\omega_{.} \equiv \sigma(1-\sigma)\left(\omega_{n}-\omega_{d}\right)
$$

is a convective mass flux, which has been defined in such a way that it is less than or equal to zero. The total turbulent flux of $\psi$ is $F_{\psi}=f_{\psi}+f_{\psi}$. 
From the "updraft" mass conservation equation (2.1) and the corresponding "downdraft" equation, we can derive an expression for $\partial \sigma / \partial t$

$$
\frac{\partial \sigma}{\partial t}+\bar{\omega} \frac{\partial}{\partial p} \sigma+\frac{\partial}{\partial p} \omega_{.}=\mu-v .
$$

By neglecting the local time rate of change and large-scale advection terms, we can simplify (2.8) to a more recognizable form:

$$
\partial \omega . / \partial p=\mu-v .
$$

According to $\left(2.9\right.$, the vertical distribution of $\omega_{0}$ is closely related to the profiles of $\mu$ and $v$.

We can also derive the conservation equation for the variance of $\psi$ that is associated with the convective circulations:

$$
\begin{aligned}
\frac{\partial}{\partial p}\left[\omega_{.}(1-2 \sigma)\left(\psi_{u}-\psi_{d}\right)\right]= & 2 g\rangle_{\psi} \frac{\partial \bar{\psi}}{\partial p}-\left[(\mu+v)-2 A \omega . / \delta p_{M}\right]\left(\psi_{\mu}-\psi_{d}\right)^{2} \\
& +2\left(\psi_{u}-\psi_{d}\right) Z_{\psi},
\end{aligned}
$$

where

$$
Z_{\varphi}=\sigma(1-\sigma)\left[g \frac{\partial}{\partial p}\left(f_{\psi, \mathrm{u}}-f_{\psi, d}\right)+\left(S_{\psi, \mathrm{u}}-S_{\psi, d}\right)\right] .
$$

In (2.10), we have neglected the local time-rate-of-change and large-scale advection terms, although we use them in our numerical model. According to (2.12), transport balances the combined effects of dissipation and production. Using conventional Reynolds averaging, we find corresponding to $(2.10)$ that

$$
\frac{\partial}{\partial p}\left(\overline{\omega^{\prime} \psi^{\prime 2}}\right)=2\left[-\overline{\mathbf{V}^{\prime} \psi^{\prime}} \cdot \nabla \bar{\psi}+g F_{\psi} \frac{\partial \bar{\psi}}{\partial p}-\varepsilon_{\psi}+\overline{\psi \bar{S}^{\prime}}{ }\right]
$$

Here $\varepsilon_{v}$ is the molecular dissipation rate. Comparison of (2.10) with (2.12) reveals a term-by-term correspondence. Note that, in (2.10), the sign of the triple correlation is determined by the value of $\sigma$. Variance is transported upward for $\sigma<1 / 2$, and downward for $\sigma>1 / 2$.

The damping factor $\sigma(1-\sigma) \tau_{\text {dis }}$, which appears in (2.10), can be simplified by noting that small eddies will be most effective at reducing the differences between the ascending and descending branches of the plumes when one of the two branches is much narrower than the other, and least effective when the two branches have the same width. On this basis, we assume that $T_{\mathrm{d}}$ is $\equiv \sigma(1-\sigma) \tau_{\mathrm{di}}$ is independent of $\sigma$. We also adopt the definition $\left(\mathrm{T}_{\mathrm{dis}}\right)^{-1} \equiv-\mathrm{A} \omega_{.} / \delta \mathrm{p}_{\mathrm{M}}$, where $\mathrm{A}$ is positive and nondimensional. This is motivated by the idea that the capacity of small eddies to dissipate the convective circulations by lateral diffusion increases as $\omega$. increases, and as the PBL depth decreases, so that $A$ should be less variable than $T_{\text {das. }}$. 
Assuming that the inflow and outflow layers do not overlap, we can eliminate $\mu$ and $v$ in (2.12), to obtain

$$
\boldsymbol{J}_{\psi} X-(1-2 \sigma) \frac{\partial \bar{y}_{\psi}}{\partial p}=-g^{-1}\left(\omega \cdot \frac{\partial \bar{\psi}}{\partial p}+Z_{\psi}\right)
$$

where

$$
\begin{gathered}
X \equiv \frac{\partial \sigma}{\partial p}+(1-\sigma-\delta) \omega_{*}^{-1} \frac{\partial \omega_{*}}{\partial p}+\frac{A}{\delta p_{M}} \\
\delta=\left\{\begin{array}{lll}
1, & \text { for } \frac{\partial \omega_{*}}{\partial p}>0 & \text { (inflow) } \\
0, & \text { for } \frac{\partial \omega_{*}}{\partial p} \leq 0 & \text { (outflow) }
\end{array}\right.
\end{gathered}
$$

The $\omega$. term of (2.14) is never negative, so it contributes to $X$ in the same sense as the positive $A$ term. It follows that $X \geq 0$ unless $\sigma$ increases strongly upward. The $\omega$. and A terms of (2.14) both arise from dissipative processes. The $\partial \sigma / \partial \mathrm{p}$ term of $(2.14)$ arises from the transport term of (2.10).

\section{Discussion} $Z_{\psi}=0$.

Several conclusions can be drawn from (2.13-14), by considering the case $S_{\psi}=0$, so that

First, if $(1-2 \sigma) / \mathrm{X}$ is small ( $\sigma$ near $1 / 2$ and strong dissipation), we get

$$
y_{w} \cong-\frac{\omega}{g X} \frac{\partial \bar{\psi}}{\partial p}
$$

This is a downgradient diffusion formula, with diffusion coefficient $-\omega_{*} /(\mathrm{gX})$.

Next, consider a layer that is well-mixed in several conservative variables $\psi_{1}, \psi_{2}$, etc. In order to satisfy (2.13) simultaneously for all of the variables we must take

$$
\begin{gathered}
X=0, \\
\sigma=1 / 2 .
\end{gathered}
$$

Since, according to (3.3), $\sigma$ is a constant, (2.14) implies that $X$ is the sum of two non-negative terms. Then (3.2) implies that each term must be zero, so that 


$$
\begin{gathered}
\omega_{.}=\text {constant, } \\
A=0 .
\end{gathered}
$$

Referring back to (2.10), we find a trivial balance in which gradient production, transport, and dissipation are all zero. (When $\sigma=1 / 2$, the variance transport by triple correlations vanishes.) reduces to

Finally, suppose that $X$ is small (weak dissipation), and that $\sigma$ is small. Then (2.14)

$$
\frac{\partial \hat{\tau}_{\psi}}{\partial p} \equiv \frac{\omega_{*}}{g} \frac{\partial \bar{\psi}}{\partial p}
$$

This balance is characteristic of the temperature and moisture budgets of tropical cumulus layers, in which "compensating subsidence" produces the convective effects on the mean profiles. Arakawa (1969) derived (3.6); it has also been discussed by Ooyama , among others. [The "detrainment" term included in cumulus parameterization theories is also present in (2.13-14); it arises from the gradient of $\omega$. in (2.14).]

For the cumulus regime, the conservation equation for $\bar{\psi}$ can often be approximated by a balance between vertical advection and convective transport, i.e.

$$
\frac{\partial \psi_{\psi}}{\partial p} \cong \frac{\bar{\omega}}{g} \frac{\partial \bar{\psi}}{\partial p} \text {. }
$$

Comparing (3.6) and (3.7), we find that

$$
\sigma=(1-\omega, / \bar{\omega}) / 2
$$

For tropical cumulus layers with large-scale rising motion, $\omega . \equiv \bar{\omega}$, so that $(3.8)$ is consistent with observations that show $\sigma \ll 1$. For the case of large-scale sinking motion, (3.8) predicts larger values of $\sigma$, again in qualitative agreement with observations.

These results show that (2.14-15) are consistent with a variety of observed balances in convective layers. They provide a dynamical interpolation between the "compensating subsidence" and "mixing length" regimes.

\section{ACKNOWLEDGEMENTS}

References have been made informally to save space. Support for this research was provided by NASA's Climate Program under Grant NAG-1-893, and by the Office of Naval Research under Contract N00014-89-J-1364. 
1 